\title{
On Exponential Stability Of Hybrid Neutral Stochastic Differential Delay Equations with Different Structures
}

\author{
Aiqing $\mathrm{Wu}^{\mathrm{a}, \mathrm{b}}$, Surong $\mathrm{You}^{\mathrm{a}, *}$, Wei Mao ${ }^{\mathrm{c}}$, Xuerong Mao ${ }^{\mathrm{d}}$, Liangjian $\mathrm{Hu}^{\mathrm{a}}$ \\ ${ }^{a}$ College of Science \\ Donghua University, Shanghai 201620, China \\ ${ }^{b}$ College of College of Materials Science and Engineering \\ Donghua University, Shanghai 201620, China \\ ${ }^{c}$ School of Mathematics and Information Technology \\ Jiangsu Second Normal University, Nanjing 210013, China \\ ${ }^{d}$ Department of Mathematics and Statistics \\ University of Strathclyde, Glasgow G1 1XH, U.K.
}

\begin{abstract}
This article discusses the problem of exponential stability for a class of hybrid neutral stochastic differential delay equations with highly nonlinear coefficients and different structures in different switching modes. In such systems, the coefficients will satisfy the local Lipschitz condition and suitable Khasminskii-types conditions. The set of switching states will be divided into two subsets. In different subsets, the coefficients will be dominated by polynomials with different degrees. By virtue of $M$-matrices and suitable Lyapunov functions dependent on coefficient structures and switching modes, some results including the existence-and-uniqueness, boundedness and exponential stability of the solution are proposed and proved.
\end{abstract}

Key words: $M$-matrix; exponential stability; hybrid neutral stochastic differential delay equation; Lyapunov function; Khasminskii-type condition

\section{Introduction}

A neutral stochastic differential delay equation(NSDDE) has been frequently applied to model a system whose future depends on the present state, the past state and its derivatives with delays. We have seen successful applications in real world, such as in population ecology([1]), processes including heat exchanges([2]), neural network([3]) and other engineering systems. A NSDEE with Markovian switching, also called a hybrid NSDDE, has also been used when a NSDDE experiences abrupt changes in its structures and coefficients caused by environmental changes and component

\footnotetext{
${ }^{*}$ Corresponding author. Tel: +86 67792086-556; Fax: +86 67792085

Email addresses: wuaiqing89@163.com (Aiqing Wu), sryou@dhu.edu.cn (Surong You), mwzy365@126.com (Wei Mao), x.mao@strath.ac.uk (Xuerong Mao), ljhu@dhu.edu.cn (Liangjian Hu)
} 
failures. There are abundant references on the theory of existence-and-uniqueness and asymptotic stability of solutions for such equations(see for example, [4]-[12] and references therein).

In most of the above mentioned works, coefficients should satisfy the local Lipschitz condition and the linear growth condition. There are many equations whose coefficients don't satisfy the linear growth condition, which leads to equations with highly nonlinear coefficients. To deal with such obstacles, new techniques have been proposed in [13]. Under suitable Khasminskii-type conditions, the asymptotic stability and boundedness of the solution can also be obtained. Thereafter, many researchers have extended the similar technique to other related equations. For hybrid NSDDEs with highly nonlinear coefficients, [14], [15] and [16] have studied moment and almost sure exponential stability of solutions. Stability and convergence of different numerical solutions of such equations have also been analyzed. [17] has discussed asymptotic boundedness and exponential stability with novel dominated conditions. [18] has studied almost sure stability with general decay rate of a hybrid NSDDE. Based on the original work [19] without neutral terms, [20] has proposed delay-dependent conditions to guarantee exponential stability. [21] has examined the boundedness and exponential stability of nonlinear hybrid neutral stochastic functional differential equations without the linear growth condition.

Let us explain the motivation of this article. In real world situations, a stochastic neutral system may experience changes in their coefficients and structures. For example, suppose there are two modes, mode $u, v$, and the system switches between two modes. Assume in mode $u$, the neutral system is $d\left(x(t)-G(x(t-\tau))=x(t)\left(a_{1}-b_{1} x^{2}(t)\right) d t+c_{1} x^{2}(t-\tau) d B_{t}\right.$, where $G(\cdot)$ is the neutral term. In mode $v$, the system becomes $d\left(x(t)-G(x(t-\tau))=x(t)\left(a_{2}-b_{2} x^{2}(t)\right) d t+c_{2} x^{2}(t-\tau) d B_{t}\right.$. Two subsystems have the same structure, but differ on their parameters. Up to now, almost all references on hybrid neutral systems have dealt with subsystems with same structures. Now consider another neutral system. The system changes from $d\left(x(t)-G(x(t-\tau))=a_{1} x(t) d t+c_{1} x(t-\tau) d B_{t}\right.$ in mode $u$ to $d\left(x(t)-G(x(t-\tau))=x(t)\left(a_{2}-b_{2} x^{2}(t)\right) d t+c_{2} x^{2}(t-\tau) d B_{t}\right.$ in mode $v$. We see different structures in two switching modes. We need new theories to study stability problem of such new system. Recently, [22] has analyzed a novel hybrid delay system, whose subsystems have different structures. This article will study the same stability problem arising from hybrid NSDDEs with highly nonlinear coefficients and different structures in different modes.

Compared to other related topics on neutral stochastic differential equations, the main contributions of this article are to take different structures in different switching modes into account in a hybrid neutral stochastic system; to propose new Khasminskii-type conditions on coefficients to prove the existence-and-uniqueness theorem of the equation; and to develop new theories on boundedness and asymptotic properties of hybrid neutral stochastic systems with different struc- 
tures. This article is arranged as follows. Some notations and concepts frequently used in this article will be introduced in section 2 . The main result will be included in section 3 , where a general criterion about coefficients is proposed to guarantee existence and uniqueness of the solution. Also the solution will be asymptotically bounded. In section 4, asymptotic properties of the solution will be stated. Some examples are given in section 5 to illustrate results obtained in this article and conclusions are made in section 6 .

\section{Preliminaries and Notations}

Throughout this article, we will use the following notations. Let $\left(\Omega, \mathcal{F},\left\{\mathcal{F}_{t}\right\}_{t \geq 0}, P\right)$ be a complete probability space with a filtration $\left\{\mathcal{F}_{t}\right\}_{t \geq 0}$ satisfying the usual conditions. Let $B(t)=$ $\left(B_{1}(t), B_{2}(t), \ldots, B_{m}(t)\right)^{T}$ be an $m$-dimensional Brownian Motion defined on the probability space. A right continuous Markov chain $r(t), t \geq 0$, takes values in a finite state space $S=\{1,2, \ldots, N\}$ with generator $\Gamma=\left(\gamma_{i j}\right)_{N \times N}$ given by

$$
P\{r(t+\Delta)=j \mid r(t)=i\}=\left\{\begin{aligned}
\gamma_{i j} \Delta+o(\Delta), & i \neq j \\
1+\gamma_{i i} \Delta+o(\Delta), & i=j
\end{aligned}\right.
$$

where $\Delta>0$, and $\gamma_{i j}>0(i \neq j)$ is the transition rate from state $i$ to $j$ with $\gamma_{i i}=-\sum_{j \neq i} \gamma_{i j}$.

We also denote by $|x|$ the Euclidean norm for $x \in \mathbb{R}^{n}$. For a matrix $A$, its trace norm is $|A|=\sqrt{\operatorname{trace}\left(A^{T} A\right)}$. Let $\mathbb{R}_{+}=[0, \infty)$ and fix $\tau>0$. The family of continuous functions $\xi:[-\tau, 0] \rightarrow \mathbb{R}^{n}$ is denoted by $C\left([-\tau, 0] ; \mathbb{R}^{n}\right)$ with the norm $\|\xi\|=\sup _{-\tau \leqslant t \leqslant 0}|\xi(t)|$. Also denote $C_{\mathcal{F}_{0}}^{b}\left([-\tau, 0] ; \mathbb{R}^{n}\right)$ the family of $\mathcal{F}_{0}$-measurable $C\left([-\tau, 0] ; \mathbb{R}^{n}\right)$-valued bounded random variables. For two real numbers $a$ and $b$, denote $a \vee b=\max (a, b)$.

For a vector $x>0$ or a matrix $A>0$, we mean that all elements in this vector or matrix are positive. A $Z$-matrix is a square matrix $A=\left(a_{i j}\right)_{N \times N}$ that has non-positive off-diagonal entries (namely $a_{i j} \leqslant 0$ for all $i \neq j$ ) and all positive diagonal entries.

Definition 2.1. A square matrix $A=\left(a_{i j}\right)_{N \times N}$ is called a nonsingular M-matrix if $A$ can be expressed in the form $A=s I-B$ with $s>\rho(B)$ while all elements of $B$ are nonnegative, where $I$ is the identity matrix and $\rho(B)$ the spectral radius of $B$.

There are many conditions which are equivalent to the statement that $A$ is a nonsingular $M$-matrix and we cite some of them for the use of this article(see e.g. [23]).

Lemma 2.1. If $A$ is a $Z$-matrix, then following statements are equivalent:

(1) $A$ is a nonsingular M-matrix. 
(2) $A$ is semi-positive; that is, there exists $x>0$ in $\mathbb{R}^{N}$ such that $A x>0$.

(3) $A^{-1}$ exists and its elements are all nonnegative.

Consider an $n$-dimensional hybrid NSDDE on $t \geq 0$ :

$$
d(x(t)-G(x(t-\tau)))=f(x(t), x(t-\tau), t, r(t)) d t+g(x(t), x(t-\tau), t, r(t)) d B(t),
$$

with the initial condition $\{x(\theta):-\tau \leqslant \theta \leqslant 0\}=\xi \in C_{\mathcal{F}_{0}}^{b}\left([-\tau, 0] ; \mathbb{R}^{n}\right)$ and $r(0)=i_{0} \in S$, where the coefficients $f: \mathbb{R}^{n} \times \mathbb{R}^{n} \times \mathbb{R}_{+} \times S \rightarrow \mathbb{R}^{n}$ and $g: \mathbb{R}^{n} \times \mathbb{R}^{n} \times \mathbb{R}_{+} \times S \rightarrow \mathbb{R}^{n}$ are Borel measurable and $G: \mathbb{R}^{n} \times S \rightarrow \mathbb{R}^{n}$ is the neutral term.

Traditional analysis on equation (1) is based on the local Lipschitz condition and the linear growth condition such as in [4]-[6]. In this article, we will focus on highly nonlinear hybrid NSDDEs whose coefficients won't satisfy the linear growth condition. We will substitute the linear growth condition for another Khasminskii-type condition. We first make the following two standing assumptions.

Assumption 2.1. For each integer $h \geq 1$, there exists a constant $K_{h}>0$ such that

$$
|f(x, y, t, i)-f(\bar{x}, \bar{y}, t, i)| \vee|g(x, y, t, i)-g(\bar{x}, \bar{y}, t, i)| \leqslant K_{h}(|x-\bar{x}|+|y-\bar{y}|)
$$

holds for those $x, y, \bar{x}, \bar{y} \in \mathbb{R}^{n}$ with $|x| \vee|\bar{x}| \vee|y| \vee|\bar{y}| \leqslant h$ and any $(t, i) \in \mathbb{R}_{+} \times S$.

Assumption 2.2. Assume that there exists a constant $\kappa \in(0,1)$ such that

$$
|G(x)-G(y)| \leqslant \kappa|x-y|
$$

holds for all $x, y \in \mathbb{R}^{n}$. We also assume that $G(0)=0$.

Immediately from Assumption 2.2, we will have $|G(x)| \leqslant \kappa|x|$.

In this article, we will focus on the asymptotic boundedness and exponential stability of equation (1).

Definition 2.2. (1) The equation is said to be asymptotically bounded in p-th moment if there is a constant $K>0$ such that

$$
\limsup _{t \rightarrow \infty} E|x(t)|^{p} \leqslant K .
$$

(2) The equation is said to be $p$-th moment exponentially stable if

$$
\limsup _{t \rightarrow \infty} \frac{\ln E|x(t)|^{p}}{t}<0 .
$$

(3) The equation is said to be almost surely exponentially stable if

$$
\limsup _{t \rightarrow \infty} \frac{\ln |x(t)|}{t}<0, \text { a.s. }
$$


It can be shown that under Assumption 2.1 and 2.2, equation (1) will have a local maximal solution([23]). In order to get the existence-and-uniqueness theorem of equation (1), additional conditions on $f$ and $g$ are necessary. We will discuss the equation with highly nonlinear coefficients. In our discussion, we will use the following notation: for a function $V(x, t, i): \mathbb{R}^{n} \times \mathbb{R}_{+} \times S \rightarrow \mathbb{R}_{+}$, define the function $L V: \mathbb{R}^{n} \times \mathbb{R}^{n} \times \mathbb{R}_{+} \times S \rightarrow \mathbb{R}$ as

$$
\begin{aligned}
L V(x, y, t, i)= & V_{t}(x-G(y), t, i)+V_{x}^{T}(x-G(y), t, i) f(x, y, t, i) \\
& +\frac{1}{2} \operatorname{trace}\left(g^{T}(x, y, t, i) V_{x x}(x-G(y), t, i) g(x, y, t, i)\right) \\
& +\sum_{j=1}^{N} \gamma_{i j} V(x-G(y), t, j)
\end{aligned}
$$

in which $V_{t}(x, t, i)=\frac{\partial V(x, t, i)}{\partial t}, V_{x}(x, t, i)=\left(\frac{\partial V(x, t, i)}{\partial x_{1}}, \frac{\partial V(x, t, i)}{\partial x_{2}}, \cdots, \frac{\partial V(x, t, i)}{\partial x_{n}}\right)^{T}$ and $V_{x x}(x, t, i)=$ $\left(\frac{\partial^{2} V(x, t, i)}{\partial x_{i} \partial x_{j}}\right)_{n \times n}$.

Some inequalities will be frequently used when we derive main results. We list them here and refer for example to $[4],[17],[18]$ and $[23]$.

Lemma 2.2. The following classical inequalities hold.

(1) For $\theta>0, p \geqslant 1$ and $a, b \geqslant 0$,

$$
(a+b)^{p} \leqslant(1+\theta)^{p-1} a^{p}+\left(1+\theta^{-1}\right)^{p-1} b^{p} .
$$

(2) For $p \geqslant 1$,

$$
(1-\kappa)^{p-1}\left(|x|^{p}-\kappa|y|^{p}\right) \leqslant|x-G(y)|^{p} \leqslant(1+\kappa)^{p-1}\left(|x|^{p}+\kappa|y|^{p}\right) .
$$

Note that the first inequality in (4) can be derived from (3) by setting $\theta=\frac{\kappa}{1-\kappa}, a=x-G(y)$ and $b=G(y)$; while the second inequality is obtained by setting $a=x, b=-G(y)$, and $\theta=\kappa$, respectively.

\section{Main Results}

In order to get the existence and uniqueness of the solution for equation (1), we need additional conditions. In this article, we will study the hybrid NSDDE whose coefficients have different forms for the problem discussed in [22]. We will make following assumptions showing nonlinearities and different structures of the neutral equation (1).

Assumption 3.1. Assume that the state space $S$ can be divided into two sub-spaces, $S_{1}=$ $\left\{1,2, \ldots, N_{1}\right\}$ and $S_{2}=\left\{N_{1}+1, N_{1}+2, \ldots, N\right\}$, with $1 \leqslant N_{1}<N$, such that coefficients have different structures in $S_{1}$ and $S_{2}$ : 
Given two constants $q$ and $p$ with $q>p \geqslant 2$, assume that for each $i \in S_{1}$ there exist constants $\alpha_{i 2} \in \mathbb{R}, \alpha_{i 1}, \alpha_{i 3} \in \mathbb{R}_{+}$such that for all $(x, y, t, i) \in \mathbb{R}^{n} \times \mathbb{R}^{n} \times \mathbb{R}_{+} \times S_{1}$,

$$
(x-G(y))^{T} f(x, y, t, i)+\frac{q-1}{2}|g(x, y, t, i)|^{2} \leqslant \alpha_{i 1}+\alpha_{i 2}|x-G(y)|^{2}+\alpha_{i 3}|y|^{2},
$$

while for each $i \in S_{2}$, there are additional positive constants $\alpha_{i 4}$ and $\alpha_{i 5}$ such that

$$
\begin{aligned}
& (x-G(y))^{T} f(x, y, t, i)+\frac{p-1}{2}|g(x, y, t, i)|^{2} \\
\leqslant & \alpha_{i 1}+\alpha_{i 2}|x-G(y)|^{2}+\alpha_{i 3}|y|^{2}-\alpha_{i 4}|x-G(y)|^{q-p+2}+\alpha_{i 5}|y|^{q-p+2} .
\end{aligned}
$$

Also assume that

$$
\mathcal{A}:=-\operatorname{diag}\left(p \alpha_{12}, \cdots, p \alpha_{N 2}\right)-\Gamma
$$

and

$$
\mathcal{D}:=-\operatorname{diag}\left(q \alpha_{12}, \cdots, q \alpha_{N_{1}, 2}\right)-\left(\gamma_{i j}\right)_{i, j \in S_{1}}
$$

are nonsingular M-matrices.

The differences in (5) and (6) reflect the different structures in $S_{1}$ and $S_{2}$. Also condition (6) has been applied, such as in [18], to analyze the stability of a neutral stochastic system with highly nonlinear coefficients. In this article, we will discuss more complicated systems with different nonlinear structures. We remark that many references, for example [8] and [21], use $|x|^{2},|y|^{2},|x|^{q-p+2}$ and $|y|^{q-p+2}$ as the dominating terms. We will use $|x|^{2},|x-G(y)|^{2},|x|^{q-p+2}$ and $|x-G(y)|^{q-p+2}$ as dominating terms for convenient application of $M$-matrices. Also we will see advantages when we calculate $L V$ for $V$ defined in (16).

Define

$$
\left(\theta_{1}, \theta_{2}, \cdots, \theta_{N}\right)^{T}=\mathcal{A}^{-1}(1,1, \cdots, 1)^{T}
$$

and

$$
\left(\eta_{1}, \eta_{2}, \cdots, \eta_{N_{1}}\right)^{T}=\mathcal{D}^{-1}(\beta, \beta, \cdots, \beta)^{T},
$$

where $\beta$ is a free positive number which will be chosen flexibly such that the conditions (12) and (13) below can be easily satisfied. In Remark 4.3 of [22], authors have given a simple way to select suitable $\beta$ such that (13) holds.

Under the assumption that $\mathcal{A}$ and $\mathcal{D}$ are nonsingular $M$-matrices, all $\theta_{i}, i \in S$ and $\eta_{j}, j \in$ $S_{1}$ are positive. Now we give the first result about the existence and uniqueness, asymptotic boundedness of the solution.

Theorem 3.1. Under Assumption 2.1,2.2 and 3.1, we also assume that

$$
\delta:=\max _{i \in S}\left(\alpha_{i 3} \theta_{i}\right)<\frac{(1-\kappa)^{p}}{(p-2)(1-\kappa)^{p}+2},
$$




$$
\hat{\beta}:=\left(\max _{i \in S_{1}} q \alpha_{i 3} \eta_{i}\right) \vee\left(\max _{i \in S_{2}} p \theta_{i} \alpha_{i 5}\right)<\frac{q(1-\kappa)^{q}}{(q-p+2)+(q-2)(1-\kappa)^{q}} \beta,
$$

and

$$
\min _{i \in S_{2}}\left(p \alpha_{i 4} \theta_{i}-\sum_{j \in S_{1}} \gamma_{i j} \eta_{j}\right) \geqslant \beta
$$

Then for any initial data $\xi \in C_{\mathcal{F}_{0}}^{b}\left([-\tau, 0] ; \mathbb{R}^{n}\right), i_{0} \in S$, we have following assertions:

(i) There exists a unique global solution $x(t)$ to the hybrid NSDDE (1).

(ii) The global solution $x(t)$ has the properties that

$$
\lim _{t \rightarrow \infty} \frac{1}{t} \int_{0}^{t} E|x(s)|^{q} d s \leqslant K_{1}
$$

and

$$
\limsup _{t \rightarrow \infty} E|x(t)|^{p} \leqslant K_{2}
$$

where $K_{1}, K_{2}$ are positive constants dependent on the initial data.

Proof. The proof will be divided into three steps.

\section{Step 1: Define $V$ function and estimate $L V$.}

Because of different structures in $S_{1}$ and $S_{2}$, we need a novel Lyapunov function related to the structure feature for further discussions.

Define a Lyapunov function $V: \mathbb{R}^{n} \times S \rightarrow \mathbb{R}_{+}$by

$$
V(x, i)= \begin{cases}\theta_{i}|x|^{p}+\eta_{i}|x|^{q}, & i \in S_{1} \\ \theta_{i}|x|^{p}, & i \in S_{2}\end{cases}
$$

where $\theta_{i}$ and $\eta_{i}$ are defined in (9) and (10), respectively. Obviously, we have

$$
c_{1}|x|^{p} \leqslant V(x, i) \leqslant c_{2}\left(|x|^{p}+|x|^{q}\right)
$$

with $c_{1}=\min _{i \in S} \theta_{i}$ and $c_{2}=\left(\max _{i \in S} \theta_{i}\right) \vee\left(\max _{i \in S_{1}} \eta_{i}\right)$.

Let $\tilde{x}_{t}=x(t)-G(x(t-\tau))$, and apply the generalized Itô formula(see, for example [23]) to $V\left(\tilde{x}_{t}, r(t)\right)$. It can be derived that

$$
V\left(\tilde{x}_{t}, r(t)\right)=V\left(\tilde{x}_{0}, i_{0}\right)+\int_{0}^{t} L V(x(s), x(s-\tau), s, r(s)) d s+M(t),
$$

where $M(t)$ is a continuous local martingale with $M(0)=0$.

Now we discuss and estimate $L V$ in $S_{1}$ and $S_{2}$, respectively. 
For $i \in S_{1}$, we have

$$
\begin{aligned}
L V(x, y, t, i)= & p \theta_{i}|x-G(y)|^{p-2}(x-G(y))^{T} f(x, y, t, i)+\frac{1}{2} p \theta_{i}|x-G(y)|^{p-2}|g(x, y, t, i)|^{2} \\
& +\frac{1}{2} p(p-2) \theta_{i}|x-G(y)|^{p-4}\left|(x-G(y))^{T} g(x, y, t, i)\right|^{2} \\
& +q \eta_{i}|x-G(y)|^{q-2}(x-G(y))^{T} f(x, y, t, i)+\frac{1}{2} q \eta_{i}|x-G(y)|^{q-2}|g(x, y, t, i)|^{2} \\
& +\frac{1}{2} q(q-2) \eta_{i}|x-G(y)|^{q-4}\left|(x-G(y))^{T} g(x, y, t, i)\right|^{2} \\
& +\sum_{j \in S} \gamma_{i j} \theta_{j}|x-G(y)|^{p}+\sum_{j \in S_{1}} \gamma_{i j} \eta_{j}|x-G(y)|^{q} .
\end{aligned}
$$

Applying the inequality $\left|x^{T} g(x, y, t, i)\right|^{2} \leqslant|x|^{2}|g(x, y, t, i)|^{2}$, (5) and (6), we get

$$
\begin{aligned}
L V(x, y, t, i) \leqslant & p \theta_{i}|x-G(y)|^{p-2}\left((x-G(y))^{T} f(x, y, t, i)+\frac{p-1}{2}|g(x, y, t, i)|^{2}\right) \\
& +q \eta_{i}|x-G(y)|^{q-2}\left((x-G(y))^{T} f(x, y, t, i)+\frac{q-1}{2}|g(x, y, t, i)|^{2}\right) \\
& +\sum_{j \in S} \gamma_{i j} \theta_{j}|x-G(y)|^{p}+\sum_{j \in S_{1}} \gamma_{i j} \eta_{j}|x-G(y)|^{q} \\
\leqslant & p \theta_{i}|x-G(y)|^{p-2}\left(\alpha_{i 1}+\alpha_{i 2}|x-G(y)|^{2}+\alpha_{i 3}|y|^{2}\right) \\
& +q \eta_{i}|x-G(y)|^{q-2}\left(\alpha_{i 1}+\alpha_{i 2}|x-G(y)|^{2}+\alpha_{i 3}|y|^{2}\right) \\
& +\sum_{j \in S} \gamma_{i j} \theta_{j}|x-G(y)|^{p}+\sum_{j \in S_{1}} \gamma_{i j} \eta_{j}|x-G(y)|^{q} .
\end{aligned}
$$

From the definition of $\theta_{i}$ and $\eta_{j},(9)$ and (10), we have

$$
p \alpha_{i 2} \theta_{i}+\sum_{j=1}^{N} \gamma_{i j} \theta_{j}=-1, i \in S,
$$

and

$$
q \alpha_{i 2} \theta_{i}+\sum_{j \in S_{1}} \gamma_{i j} \eta_{j}=-\beta, i \in S_{1},
$$

and then consequently, for any $i \in S_{1}$,

$$
\begin{aligned}
L V(x, y, t, i) \leqslant & p \theta_{i} \alpha_{i 1}|x-G(y)|^{p-2}-|x-G(y)|^{p}+p \theta_{i} \alpha_{i 3}\left(\frac{p-2}{p}|x-G(y)|^{p}+\frac{2}{p}|y|^{p}\right) \\
& +q \eta_{i} \alpha_{i 1}|x-G(y)|^{q-2}-\beta|x-G(y)|^{q}+q \eta_{i} \alpha_{i 3}\left(\frac{q-2}{q}|x-G(y)|^{q}+\frac{2}{q}|y|^{q}\right) \\
= & p \theta_{i} \alpha_{i 1}|x-G(y)|^{p-2}-\left(1-(p-2) \theta_{i} \alpha_{i 3}\right)|x-G(y)|^{p}+2 \theta_{i} \alpha_{i 3}|y|^{p} \\
& +q \eta_{i} \alpha_{i 1}|x-G(y)|^{q-2}-\left(\beta-(q-2) \eta_{i} \alpha_{i 3}\right)|x-G(y)|^{q}+2 \eta_{i} \alpha_{i 3}|y|^{q} .
\end{aligned}
$$

Similarly, for any $i \in S_{2}, L V(x, y, t, i)$ will satisfy

$$
\begin{aligned}
L V(x, y, t, i) \leqslant & p \theta_{i} \alpha_{i 1}|x-G(y)|^{p-2}-\left(1-(p-2) \theta_{i} \alpha_{i 3}\right)|x-G(y)|^{p}+2 \theta_{i} \alpha_{i 3}|y|^{p} \\
& -\left(\beta-\frac{p(p-2)}{q} \theta_{i} \alpha_{i 5}\right)|x-G(y)|^{q}+\frac{p(q-p+2)}{q} \theta_{i} \alpha_{i 5}|y|^{q} .
\end{aligned}
$$

Applying $\delta$ and $\hat{\beta}$ defined in (11) and (12) respectively, we can get that for any $i \in S$,

$$
\begin{aligned}
L V(x, y, t, i) \leqslant & c_{3}\left(|x-G(y)|^{p-2}+|x-G(y)|^{q-2}\right)-(1-(p-2) \delta)|x-G(y)|^{p}+2 \delta|y|^{p} \\
& -\left(\beta-\frac{q-2}{q} \hat{\beta}\right)|x-G(y)|^{q}+\frac{q-p+2}{q} \hat{\beta}|y|^{q},
\end{aligned}
$$


where $c_{3}:=\left(\max _{i \in S} p \theta_{i} \alpha_{i 1}\right) \vee\left(\max _{i \in S_{1}} q \eta_{i} \alpha_{i 1}\right)$.

For convenience, introduce $\beta_{2}:=\frac{q-p+2}{q} \hat{\beta}$. From the condition (12), there exists some $\alpha_{0} \in$ $(0,1)$ such that

$$
\beta_{1}:=\alpha_{0}\left(\beta-\frac{q-2}{q} \hat{\beta}\right)>\frac{1}{(1-\kappa)^{q}} \beta_{2}
$$

holds.

To see this, it can be derived from (12) that

$$
\beta-\frac{q-2}{q} \hat{\beta}>\left(1-\frac{(q-2)(1-\kappa)^{q}}{(q-p+2)+(q-2)(1-\kappa)^{q}}\right) \beta>0
$$

and

$$
\beta-\frac{q-2}{q} \hat{\beta}-\frac{1}{(1-\kappa)^{q}} \beta_{2}=\frac{q(1-\kappa)^{q} \beta-\left((q-2)(1-\kappa)^{q}+(q-p+2)\right) \hat{\beta}}{q(1-\kappa)^{q}}>0
$$

hold, so that any $\alpha_{0} \in\left(\frac{(q-p+2) \hat{\beta}(1-\kappa)^{-q}}{q \beta-(q-2) \hat{\beta}}, 1\right)$ makes sense.

Then (21) can be rewritten as

$$
\begin{aligned}
L V(x, y, t, i) \leqslant & c_{3}\left(|x-G(y)|^{p-2}+|x-G(y)|^{q-2}\right)-\frac{1-\alpha_{0}}{\alpha_{0}} \beta_{1}|x-G(y)|^{q} \\
& -(1-(p-2) \delta)|x-G(y)|^{p}+2 \delta|y|^{p}-\beta_{1}|x-G(y)|^{q}+\beta_{2}|y|^{q} .
\end{aligned}
$$

Note that for positive $c_{3}>0$ and $\beta_{1}>0$, the function

$$
\varphi(u)=c_{3}\left(u^{p-2}+u^{q-2}\right)-\frac{1-\alpha_{0}}{\alpha_{0}} \beta_{1} u^{q}
$$

will have a finite supremum value over $[0,+\infty)$, denoted by $c_{4}:=\sup _{u \in[0,+\infty)} \varphi(u)<+\infty$. Hence,

$$
L V(x, y, t, i) \leqslant c_{4}-(1-(p-2) \delta)|x-G(y)|^{p}+2 \delta|y|^{p}-\beta_{1}|x-G(y)|^{q}+\beta_{2}|y|^{q} .
$$

Moreover, by (4), we have the final estimation for $L V$,

$$
\begin{aligned}
L V(x, y, t, i) \leqslant & c_{4}-(1-\delta(p-2))(1-\kappa)^{p-1}|x|^{p}+\left(2 \delta+(1-\delta(p-2)) \kappa(1-\kappa)^{p-1}\right)|y|^{p} \\
& -\beta_{1}(1-\kappa)^{q-1}|x|^{q}+\left(\beta_{2}+\left(\beta_{1}+\beta_{2}\right)(1-\kappa)^{q-1} \kappa\right)|y|^{q} .
\end{aligned}
$$

Step 2: Prove the existence and uniqueness of the solution.

We will prove the existence and uniqueness of the global solution of equation (1) on $t \in$ $[-\tau, \infty)$. By Assumption 2.1 and 2.2, there exists a unique maximal local solution $x(t)$ on $t \in$ $\left[-\tau, \sigma_{\infty}\right)$ where $\sigma_{\infty}$ is the explosion time $([23])$. Therefore we need to prove $\sigma_{\infty}=\infty$. Suppose $\gamma_{0}$ be a sufficiently large positive integer such that $|\xi|<\gamma_{0}$. For each $\gamma \geqslant \gamma_{0}$, define the stopping time $\tau_{\gamma}$ by

$$
\tau_{\gamma}=\inf \{t \geqslant 0:|x(t)| \geqslant \gamma\} .
$$

It is easy to see that $\tau_{\gamma}$ is increasing. Define $\tau_{\infty}:=\lim _{\gamma \rightarrow \infty} \tau_{\gamma} \leqslant \sigma_{\infty}$. We will show that $\tau_{\infty}=\infty$. 
Now by taking expectation to the localized form $V\left(\tilde{x}\left(t \wedge \tau_{\gamma}\right), r\left(t \wedge \tau_{\gamma}\right)\right)$, it can be derived that

$$
\begin{aligned}
& E V\left(\tilde{x}_{t \wedge \tau_{\gamma}}, r\left(t \wedge \tau_{\gamma}\right)\right) \\
= & E V\left(\tilde{x}_{0}, r_{0}\right)+E \int_{0}^{t \wedge \tau_{\gamma}} L V(x(s), x(s-\tau), s, r(s)) d s \\
\leqslant & E V\left(\tilde{x}_{0}, r_{0}\right)+c_{4}\left(t \wedge \tau_{\gamma}\right)-(1-\delta(p-2))(1-\kappa)^{p-1} E \int_{0}^{t \wedge \tau_{\gamma}}|x(s)|^{p} d s \\
& +\left(2 \delta+(1-\delta(p-2)) \kappa(1-\kappa)^{p-1}\right) E \int_{0}^{t \wedge \tau_{\gamma}}|x(s-\tau)|^{p} d s \\
& -\beta_{1}(1-\kappa)^{q-1} E \int_{0}^{t \wedge \tau_{\gamma}}|x(s)|^{q} d s+\left(\beta_{2}+\beta_{1}(1-\kappa)^{q-1} \kappa\right) E \int_{0}^{t \wedge \tau_{\gamma}}|x(s-\tau)|^{q} d s .
\end{aligned}
$$

For any $p>0$, we have the fact that

$$
E \int_{0}^{t \wedge \tau_{\gamma}}|x(s-\tau)|^{p} d s \leqslant E \int_{-\tau}^{0}|x(s)|^{p} d s+E \int_{0}^{t \wedge \tau_{\gamma}}|x(s)|^{p} d s .
$$

Consequently, by applying the inequality $(17),(27)$ can be reduced to

$$
\begin{aligned}
c_{1} E\left|\tilde{x}_{t \wedge \tau_{\gamma}}\right|^{p} \leqslant & K_{3}+c_{4} t+\left(2 \delta-(1-\delta(p-2))(1-\kappa)^{p}\right) E \int_{0}^{t \wedge \tau_{\gamma}}|x(s)|^{p} d s \\
& +\left(\beta_{2}-\beta_{1}(1-\kappa)^{q}\right) E \int_{0}^{t \wedge \tau_{\gamma}}|x(s)|^{q} d s,
\end{aligned}
$$

where

$$
\begin{aligned}
K_{3}= & c_{2}\left(2^{p-1}(1+\kappa)^{p} E\|\xi\|^{p}+2^{q-1}(1+\kappa)^{q} E\|\xi\|^{q}\right) \\
& +\left(2 \delta+(1-\delta(p-2))(1-\kappa)^{p-1} \kappa\right) E \int_{-\tau}^{0}|x(s)|^{p} d s \\
& +\left(\beta_{2}+\beta_{1}(1-\kappa)^{q-1} \kappa\right) E \int_{-\tau}^{0}|x(s)|^{q} d s .
\end{aligned}
$$

By conditions (11) and (22), we can get that

$$
c_{1} E\left|\tilde{x}_{t \wedge \tau_{\gamma}}\right|^{p} \leqslant K_{3}+c_{4} t
$$

Applying inequality (4), we have

$$
(1-\kappa)^{p-1} E\left|x\left(t \wedge \tau_{\gamma}\right)\right|^{p} \leqslant E\left|\tilde{x}_{t \wedge \tau_{\gamma}}\right|^{p}+\kappa(1-\kappa)^{p-1} E\left|x\left(t \wedge \tau_{\gamma}-\tau\right)\right|^{p} .
$$

From (30), it can be derived that

$$
\begin{aligned}
c_{1}(1-\kappa)^{p-1} \sup _{0 \leqslant s \leqslant t} E\left|x\left(s \wedge \tau_{\gamma}\right)\right|^{p} & \leqslant K_{3}+c_{4} t+c_{1} \kappa(1-\kappa)^{p-1} \sup _{0 \leqslant s \leqslant t} E\left|x\left(s \wedge \tau_{\gamma}-\tau\right)\right|^{p} \\
& \leqslant K_{3}^{\prime}+c_{4} t+c_{1} \kappa(1-\kappa)^{p-1} \sup _{0 \leqslant s \leqslant t} E\left|x\left(s \wedge \tau_{\gamma}\right)\right|^{p}
\end{aligned}
$$

and then

$$
c_{1}(1-\kappa)^{p} E\left|x\left(t \wedge \tau_{\gamma}\right)\right|^{p} \leqslant K_{3}^{\prime}+c_{4} t,
$$

where $K_{3}^{\prime}=K_{3}+\kappa(1-\kappa)^{p-1} c_{1} \sup _{-\tau \leqslant s \leqslant 0} E|x(s)|^{p}$. 
Consequently, we get

$$
c_{1}(1-\kappa)^{p} \gamma^{p} P\left(\tau_{\gamma} \leqslant t\right) \leqslant K_{3}^{\prime}+c_{4} t .
$$

As $\gamma \rightarrow \infty$, we can see that for any $t, \lim _{\gamma \rightarrow \infty} P\left(\tau_{\gamma} \leqslant t\right)=0$, which means that for any $t, \tau_{\infty} \geq t$, and then $\tau_{\infty}=\infty$. Now we have proved the first assertion.

\section{Step 3: Show properties of the solution.}

From (30), it can be easily shown that

$$
c_{1} E\left|\tilde{x}_{t \wedge \tau_{\gamma}}\right|^{p} \leqslant K_{3}+c_{4} t+\left(\beta_{2}-\beta_{1}(1-\kappa)^{q}\right) E \int_{0}^{t \wedge \tau_{\gamma}}|x(s)|^{q} d s,
$$

so that

$$
\left(\beta_{1}(1-\kappa)^{q}-\beta_{2}\right) E \int_{0}^{t \wedge \tau_{\gamma}}|x(s)|^{q} d s \leqslant K_{3}+c_{4} t .
$$

By the fact that $\beta_{1}(1-\kappa)^{q}>\beta_{2}$, if we let $\gamma \rightarrow \infty$ and apply the monotone convergence theorem, it then follows, if the limit as $t \rightarrow \infty$ is then taken, that the second desired assertion (14), is obtained:

$$
\limsup _{t \rightarrow \infty} \frac{1}{t} \int_{0}^{t} E|x(s)|^{q} d s \leqslant \frac{c_{4}}{\beta_{1}(1-\kappa)^{q}-\beta_{2}}=: K_{1} .
$$

In order to prove the last assertion (15), define two functions $F_{1}(\varepsilon), F_{2}(\varepsilon): \mathbb{R}^{+} \rightarrow \mathbb{R}$ as

$$
\begin{gathered}
F_{1}(\varepsilon):=\beta_{1}(1-\kappa)^{q-1}\left(\mathrm{e}^{\varepsilon \tau} \kappa-1\right)+\beta_{2} \mathrm{e}^{\varepsilon \tau}+\varepsilon c_{2}(1+\kappa)^{q-1}\left(\mathrm{e}^{\varepsilon \tau} \kappa+1\right), \\
F_{2}(\varepsilon):=\varepsilon c_{2}(1+\kappa)^{p-1}\left(\mathrm{e}^{\varepsilon \tau} \kappa+1\right)+\mathrm{e}^{\varepsilon \tau}\left(2 \delta+(1-\delta(p-2))(1-\kappa)^{p-1} \kappa\right)-(1-\delta(p-2))(1-\kappa)^{p-1} .
\end{gathered}
$$

Obviously, $F_{1}(\varepsilon)$ is strictly increasing in $\varepsilon$, and $\lim _{\varepsilon \rightarrow+\infty} F_{1}(\varepsilon)=+\infty$. Meanwhile, by $(22), F_{1}(0)=$ $-\beta_{1}(1-\kappa)^{q}+\beta_{2}<0$, so that there exists a unique $\varepsilon^{*}>0$ such that $F_{1}\left(\varepsilon^{*}\right)=0$, and for any $\varepsilon<\varepsilon^{*}, F_{1}(\varepsilon)<0$.

Fix some $\varepsilon_{0}<\varepsilon^{*}$ with $F_{1}\left(\varepsilon_{0}\right)<0$ and define another function $H(u): \mathbb{R}^{+} \rightarrow \mathbb{R}$ as

$$
H(u)=c_{4}+F_{2}\left(\varepsilon_{0}\right) u^{p}+F_{1}\left(\varepsilon_{0}\right) u^{q} .
$$

As $q>p$ and $F_{1}\left(\varepsilon_{0}\right)<0$, we can get a finite supremum of $H(u)$, denoted as $c_{5}:=\sup _{u \geqslant 0} H(u)<\infty$.

Applying the generalized Itô formula to $\mathrm{e}^{\varepsilon_{0} t} V\left(\tilde{x}_{t}, r(t)\right)$, it can be derived that

$$
\mathrm{e}^{\varepsilon_{0} t} V\left(\tilde{x}_{t}, r(t)\right)=V\left(\tilde{x}_{0}, i_{0}\right)+\int_{0}^{t} \mathrm{e}^{\varepsilon_{0} s}\left(\varepsilon_{0} V\left(\tilde{x}_{s}, r(s)\right)+L V(x(s), x(s-\tau), s, r(s))\right) d s+M_{1}(t)
$$

where $M_{1}(t)$ is another continuous local martingale with $M_{1}(0)=0$ on $t \geqslant 0$. 
Just as in the discussion above, taking expectation to the localized form $\mathrm{e}^{\varepsilon_{0}\left(t \wedge \tau_{\gamma}\right)} V\left(\tilde{x}_{t \wedge \tau_{\gamma}}, r(t \wedge\right.$ $\left.\tau_{\gamma}\right)$ ), with the stopping times $\tau_{\gamma}$ defined in (26), we have

$$
\begin{aligned}
& E\left(\mathrm{e}^{\varepsilon_{0}\left(t \wedge \tau_{\gamma}\right)} V\left(\tilde{x}_{t \wedge \tau_{\gamma}}, r\left(t \wedge \tau_{\gamma}\right)\right)\right) \\
= & E V\left(\tilde{x}_{0}, i_{0}\right)+E \int_{0}^{t \wedge \tau_{\gamma}} \mathrm{e}^{\varepsilon_{0} s}\left(\varepsilon_{0} V\left(\tilde{x}_{s}, r(s)\right)+L V(x(s), x(s-\tau), s, r(s))\right) d s .
\end{aligned}
$$

Substituting (25) into the above equation, and using the inequality (4), it can be derived that

$$
\begin{aligned}
& E\left(\mathrm{e}^{\varepsilon_{0}\left(t \wedge \tau_{\gamma}\right)} V\left(\tilde{x}_{t \wedge \tau_{\gamma}}, r\left(t \wedge \tau_{\gamma}\right)\right)\right) \\
\leqslant & c_{2}\left(2^{p-1}(1+\kappa)^{p} E\|\xi\|^{p}+2^{q-1}(1+\kappa)^{q} E\|\xi\|^{q}\right)+\frac{c_{4}}{\varepsilon_{0}} \mathrm{e}^{\varepsilon_{0}\left(t \wedge \tau_{\gamma}\right)} \\
& +E \int_{0}^{t \wedge \tau_{\gamma}} \mathrm{e}^{\varepsilon_{0} s}\left\{\varepsilon_{0} c_{2}\left(|x(s)-G(x(s-\tau))|^{p}+|x(s)-G(x(s-\tau))|^{q}\right)\right. \\
& -\left((1-\delta(p-2))(1-\kappa)^{p-1}\right)|x(s)|^{p}+\left(2 \delta+(1-\delta(p-2))(1-\kappa)^{p-1} \kappa\right)|x(s-\tau)|^{p} \\
& \left.-\beta_{1}(1-\kappa)^{q-1}|x(s)|^{q}+\left(\beta_{2}+\beta_{1}(1-\kappa)^{q-1} \kappa\right)|x(s-\tau)|^{q}\right\} d s \\
\leqslant & K_{4}+\frac{c_{4}}{\varepsilon_{0}} \mathrm{e}^{\varepsilon_{0} t}+F_{2}\left(\varepsilon_{0}\right) E \int_{0}^{t \wedge \tau_{\gamma}} \mathrm{e}^{\varepsilon_{0} s}|x(s)|^{p} d s+F_{1}\left(\varepsilon_{0}\right) E \int_{0}^{t \wedge \tau_{\gamma}} \mathrm{e}^{\varepsilon_{0} s}|x(s)|^{q} d s,
\end{aligned}
$$

where

$$
\begin{aligned}
K_{4}= & c_{2}\left(2^{p-1}(1+\kappa)^{p} E\|\xi\|^{p}+2^{q-1}(1+\kappa)^{q} E\|\xi\|^{q}\right) \\
& +\left(\varepsilon_{0} c_{2}(1+\kappa)^{p-1} \kappa+2 \delta+(1-\delta(p-2))(1-\kappa)^{p-1} \kappa\right) \mathrm{e}^{\varepsilon_{0} \tau} E \int_{-\tau}^{0}|x(s)|^{p} d s \\
& +\left(\varepsilon_{0} c_{2}(1+\kappa)^{q-1} \kappa+\beta_{2}+\left(\beta_{1}+\beta_{2}\right)(1-\kappa)^{q-1} \kappa\right) \mathrm{e}^{\varepsilon_{0} \tau} E \int_{-\tau}^{0}|x(s)|^{q} d s .
\end{aligned}
$$

Taking $\gamma \rightarrow+\infty$ and applying (17), we will obtain

$$
c_{1} \mathrm{e}^{\varepsilon_{0} t} E\left|\tilde{x}_{t}\right|^{p} \leqslant K_{4}+E \int_{0}^{t} \mathrm{e}^{\varepsilon_{0} s} H(|x(s)|) d s \leqslant K_{4}+\frac{c_{5}}{\varepsilon_{0}}\left(\mathrm{e}^{\varepsilon_{0} t}-1\right) .
$$

Using the inequality (3) by taking $\theta=\theta_{0}>\left(\kappa^{\frac{-p}{p-1}}-1\right)^{-1}$, we can get that

$$
\begin{aligned}
E|x(t)|^{p} & \leqslant\left(1+\theta_{0}\right)^{p-1} E\left|\tilde{x}_{t}\right|^{p}+\left(1+\theta_{0}^{-1}\right)^{p-1} E|G(x(t-\tau))|^{p} \\
& \leqslant\left(1+\theta_{0}\right)^{p-1}\left(\frac{K_{4}}{c_{1}} \mathrm{e}^{-\varepsilon_{0} t}+\frac{c_{5}}{c_{1} \varepsilon_{0}}\right)+\left(1+\theta_{0}^{-1}\right)^{p-1} E|G(x(t-\tau))|^{p} \\
& \leqslant\left(1+\theta_{0}\right)^{p-1}\left(\frac{K_{4}}{c_{1}} \mathrm{e}^{-\varepsilon_{0} t}+\frac{c_{5}}{c_{1} \varepsilon_{0}}\right)+\kappa^{p}\left(1+\theta_{0}^{-1}\right)^{p-1} E|x(t-\tau)|^{p}
\end{aligned}
$$

and consequently, for any $t \geq 0$,

$$
\sup _{0 \leqslant s \leqslant t} E|x(s)|^{p} \leqslant\left(1+\theta_{0}\right)^{p-1}\left(\frac{K_{4}}{c_{1}} \mathrm{e}^{-\varepsilon_{0} t}+\frac{c_{5}}{c_{1} \varepsilon_{0}}\right)+\kappa^{p}\left(1+\theta_{0}^{-1}\right)^{p-1}\left(\sup _{-\tau \leqslant s \leqslant 0} E|x(s)|^{p}+\sup _{0 \leqslant s \leqslant t} E|x(s)|^{p}\right)
$$

or equivalently, by the fact that $\left(1+\theta_{0}^{-1}\right)^{p-1} \kappa^{p}<1$,

$$
\sup _{0 \leqslant s \leqslant t} E|x(s)|^{p} \leqslant \frac{(1+\theta)^{p-1}\left(\frac{K_{4}}{c_{1}} \mathrm{e}^{-\varepsilon_{0} t}+\frac{c_{5}}{c_{1} \varepsilon_{0}}\right)+\kappa^{p}\left(1+\theta_{0}^{-1}\right)^{p-1} E\|\xi\|^{p}}{1-\left(1+\theta_{0}^{-1}\right)^{p-1} \kappa^{p}},
$$


which implies the assertion (15):

$$
\limsup _{t \rightarrow \infty} E|x(t)|^{p} \leqslant \frac{c_{5}\left(1+\theta_{0}\right)^{p-1}+c_{1} \varepsilon_{0} \kappa^{p}\left(1+\theta_{0}^{-1}\right)^{p-1} E\|\xi\|^{p}}{c_{1} \varepsilon_{0}\left(1-\left(1+\theta_{0}^{-1}\right)^{p-1} \kappa^{p}\right)}=: K_{2}
$$

This completes the proof.

\section{Asymptotic properties of the solution}

In this section, we will discuss the asymptotic properties of the solution for equation (1). Assume $f(0,0, t, i)=g(0,0, t, i)=0$ for any $(t, i) \in \mathbb{R}_{+} \times S$, so that the equation (1) has a trivial solution $x(t)=0$ with the initial condition $\xi=0$, and then Assumption 3.1 can be simplified to let $\alpha_{i 1}=0$ for any $i \in S$. Firstly, we have following theorem on $H_{\infty}$ stability of the solution.

Theorem 4.1. Assume that all conditions in Theorem 3.1 hold, and for any $i \in S, \alpha_{i 1}=0$, then for any initial data $\xi \in C\left([-\tau, 0] ; \mathbb{R}^{n}\right)$ and $i_{0} \in S$, the unique global solution $x(t)$ of equation (1) satisfies

$$
\int_{0}^{\infty} E|x(t)|^{q} d t<\infty \text { and } \int_{0}^{\infty} E\left|\tilde{x}_{t}\right|^{q} d t<\infty
$$

Proof. When $\alpha_{i 1}=0$ holds for any $i \in S, c_{3}$ in (21) will be zero. Applying the same argument and notations as in Theorem 3.1, we can get that for any $i \in S$,

$$
\begin{aligned}
L V(x, y, t, i) \leqslant & -(1-\delta(p-2))(1-\kappa)^{p-1}|x|^{p}+\left(2 \delta+(1-\delta(p-2))(1-\kappa)^{p-1} \kappa\right)|y|^{p} \\
& -\left(\beta-\frac{q-2}{q} \hat{\beta}\right)(1-\kappa)^{q-1}|x|^{q}+\left(\frac{q-p+2}{q} \hat{\beta}+\left(\beta-\frac{q-2}{q} \hat{\beta}\right) \kappa(1-\kappa)^{q-1}\right)|y|^{q} .
\end{aligned}
$$

Applying the generalized Itô's formula and the localized argument as in Theorem 3.1, it can be shown that for any $t>0$,

$$
\begin{aligned}
c_{1} E\left(\left|\tilde{x}_{t}\right|^{p}\right) \leq & K_{6}-\left((1-\kappa)^{p}-\left(2+(p-2)(1-\kappa)^{p}\right) \delta\right) E \int_{0}^{t}|x(s)|^{p} d s \\
& -\left(q(1-\kappa)^{q} \beta-\left((q-p+2)+(q-2)(1-\kappa)^{q}\right) \hat{\beta}\right) E \int_{0}^{t}|x(s)|^{q} d s
\end{aligned}
$$

where

$$
\begin{aligned}
K_{6}= & c_{2}\left(2^{p-1}(1+\kappa)^{p}\|\xi\|^{p}+2^{q-1}(1+\kappa)^{q}\|\xi\|^{q}\right) \\
& +\left((1-\delta(p-2))(1-\kappa)^{p-1} \kappa+2 \delta\right) \int_{-\tau}^{0} E|x(s)|^{p} d s \\
& +\left(\frac{q-p+2}{q} \hat{\beta}+\left(\beta-\frac{q-2}{q} \hat{\beta}\right) \kappa(1-\kappa)^{q-1}\right) \int_{-\tau}^{0} E|x(s)|^{q} d s .
\end{aligned}
$$

By condition (11) and (12), we have $(1-\kappa)^{p}-\left(2+(p-2)(1-\kappa)^{p}\right) \delta>0$ and $q(1-\kappa)^{q} \beta-$ $\left((q-p+2)+(q-2)(1-\kappa)^{q}\right) \hat{\beta}>0$, and consequently,

$$
E \int_{0}^{t}|x(s)|^{q} d s \leq \frac{K_{6}}{q(1-\kappa)^{q} \beta-\left((q-p+2)+(q-2)(1-\kappa)^{q}\right) \hat{\beta}} .
$$


The assertion $\int_{0}^{\infty} E|x(t)|^{q} d t<\infty$ is then verified by letting $t \rightarrow \infty$ and using Fubini's theorem. Furthermore, for any $t>0$ it is true that

$$
\begin{aligned}
\int_{0}^{t}\left|\tilde{x}_{s}\right|^{q} d s & \leq 2^{q-1}\left(\int_{0}^{t}|x(s)|^{q} d s+\kappa^{q} \int_{0}^{t}|x(s-\tau)|^{q} d s\right) \\
& \leq 2^{q-1} \kappa^{q} \int_{-\tau}^{0}|x(s)|^{q} d s+2^{q-1}\left(1+\kappa^{q}\right) \int_{0}^{t}|x(s)|^{q} d s
\end{aligned}
$$

which can be used to derive the second $H_{\infty}$ result, namely that $\int_{0}^{\infty} E\left|\tilde{x}_{t}\right|^{q} d t<\infty$.

Generally, we can't obtain $\lim _{t \rightarrow \infty} E|x(t)|^{q}=0$ from (42). In order to get such an asymptotic property, we need an additional condition as in (44) below.

Theorem 4.2. Assume all conditions in Theorem 4.1 hold. If there exists a positive constant $K_{0}$ such that for any $(x, y, t, i) \in \mathbb{R}^{n} \times \mathbb{R}^{n} \times \mathbb{R}_{+} \times S$,

$$
(x-D(y))^{T} f(x, y, t, i)+\frac{q-1}{2}|g(x, y, t, i)|^{2} \leq K_{0}\left(|x-D(y)|^{2}+|y|^{2}\right)
$$

then for any initial data $\xi \in C\left([-\tau, 0] ; \mathbb{R}^{n}\right)$, the equation is also asymptotically stable in $L^{q}$ :

$$
\lim _{t \rightarrow \infty} E|x(t)|^{q}=0
$$

Proof. Firstly, we will show

$$
\lim _{t \rightarrow \infty} E\left|\tilde{x}_{t}\right|^{q}=0
$$

Suppose to the contrary that $\lim _{t \rightarrow \infty} E\left|\tilde{x}_{t}\right|^{q} \neq 0$. Then there exists an $\varepsilon>0$ and a sequence $\left\{t_{k}\right\}_{k \geqslant 1}$ such that $t_{1} \geqslant 2 \tau, t_{k+1}>t_{k}+2 \tau$, and for any $k \geqslant 1, E\left|\tilde{x}_{t_{k}}\right|^{q} \geqslant 2 \varepsilon$.

From Theorem 4.1, we have

$$
\sum_{k=1}^{\infty} \int_{t_{k}-2 \tau}^{t_{k}} E|x(s)|^{q} d s \leqslant \int_{0}^{\infty} E|x(s)|^{q} d s<\infty
$$

which means

$$
\lim _{k \rightarrow \infty} \int_{t_{k}-2 \tau}^{t_{k}} E|x(s)|^{q} d s=0 .
$$

So there must be some $k_{0}>0$ such that

$$
\int_{t_{k}-2 \tau}^{t_{k}} E|x(s)|^{q} d s<\frac{\varepsilon}{2 q K_{0}(1+\kappa)^{q-1}}
$$

holds for $k \geqslant k_{0}$. 
On the other hand, for any $k \geqslant k_{0}$ and $t \in\left[t_{k}-\tau, t_{k}\right]$, it is true that

$$
\begin{aligned}
& E\left|\tilde{x}_{t_{k}}\right|^{q}-E\left|\tilde{x}_{t}\right|^{q} \\
\leqslant & E \int_{t}^{t_{k}} q\left|\tilde{x}_{s}\right|^{q-2}\left(\tilde{x}_{s}^{T} f(x(s), x(s-\tau), s, r(s))+\frac{q-1}{2}|g(x(s), x(s-\tau), s, r(s))|^{2}\right) d s \\
\leqslant & K_{0} E \int_{t}^{t_{k}} q\left|\tilde{x}_{s}\right|^{q-2}\left(\left|\tilde{x}_{s}\right|^{2}+|x(s-\tau)|^{2}\right) d s \\
\leqslant & K_{0} E \int_{t}^{t_{k}}\left(2(q-1)\left|\tilde{x}_{s}\right|^{q}+2|x(s-\tau)|^{q}\right) d s \\
\leqslant & 2 q K_{0}(1+\kappa)^{q-1} E \int_{t}^{t_{k}}\left(|x(s)|^{q}+|x(s-\tau)|^{q}\right) d s \\
\leqslant & 2 q K_{0}(1+\kappa)^{q-1} E \int_{t_{k}-2 \tau}^{t_{k}}|x(s)|^{q} d s \\
\leqslant & \varepsilon .
\end{aligned}
$$

So we get $E\left|\tilde{x}_{t}\right|^{q} \geqslant E\left|\tilde{x}_{t_{k}}\right|-\varepsilon \geqslant \varepsilon$, and then

$$
\int_{0}^{\infty} E\left|\tilde{x}_{t}\right|^{q} d t \geqslant \sum_{k=1}^{\infty} \int_{t_{k}}^{t_{k+1}} E\left|\tilde{x}_{t}\right|^{q} d t>\sum_{k=1}^{\infty} 2 \tau \varepsilon=\infty
$$

which contradicts the second result in (42).

From (4), we have

$$
E|x(t)|^{q} \leqslant \frac{1}{(1-\kappa)^{q-1}} E|\tilde{x}(t)|^{q}+\kappa E|x(t-\tau)|^{q} .
$$

Taking $t \rightarrow \infty$ on both sides gives

$$
\limsup _{t \rightarrow \infty} E|x(t)|^{q} \leqslant \kappa \limsup _{t \rightarrow \infty} E|x(t)|^{q},
$$

and then (45) holds as required.

In Theorem 4.2, the asymptotic stability of the $q$-th moment of the solution has been proved under the stronger condition (44). In the next theorem, we will show the exponential stability of the $p$-th moment of the solution without (44).

Theorem 4.3. Assume that all conditions in Theorem 3.1 hold. Define

$$
\lambda_{0}:=\min \left\{\lambda_{1}, \lambda_{2},-\frac{\ln \kappa}{\tau}\right\},
$$

where $\lambda_{1}, \lambda_{2}$ are unique positive roots of the equations $J_{1}(\lambda)=0, J_{2}(\lambda)=0$ respectively, where

$$
\begin{gathered}
J_{1}(\lambda)=\lambda c_{2}(1+\kappa)^{p-1}\left(\mathrm{e}^{\lambda \tau} \kappa+1\right)+2 \delta \mathrm{e}^{\lambda \tau}-(1-\delta(p-2))(1-\kappa)^{p-1}\left(1-\mathrm{e}^{\lambda \tau} \kappa\right), \\
J_{2}(\lambda)=\lambda c_{2}(1+\kappa)^{q-1}\left(\mathrm{e}^{\lambda \tau} \kappa+1\right)+\frac{q-p+2}{q} \hat{\beta} \mathrm{e}^{\lambda \tau}-\left(\beta-\frac{q-2}{q} \hat{\beta}\right)(1-\kappa)^{q-1}\left(1-\mathrm{e}^{\lambda \tau} \kappa\right)
\end{gathered}
$$

respectively. If $\alpha_{i 1}=0$ hold for all $i \in S$, then for any initial data $\xi \in C\left([-\tau, 0] ; \mathbb{R}^{n}\right)$ and $i_{0} \in S$, the unique global solution $x(t)$ of equation (1) satisfies

$$
\limsup _{t \rightarrow \infty} \frac{1}{t} \log \left(E|x(t)|^{p}\right) \leqslant-\lambda_{0}
$$

and

$$
\limsup _{t \rightarrow \infty} \frac{1}{t} \log (|x(t)|) \leqslant-\frac{\lambda_{0}}{p}
$$


Proof. Firstly, we show that such $\lambda_{1}$ and $\lambda_{2}$ in (47) exist and $\lambda_{0}>0$. From (11) and (22), direct calculation gives

$$
J_{1}(0)=2 \delta-(1-\delta(p-2))(1-\kappa)^{p}<0 \text { and } J_{2}(0)=\frac{q-p+2}{q} \hat{\beta}-\left(\beta-\frac{q-2}{q} \hat{\beta}\right)(1-\kappa)^{q}<0 .
$$

Clearly, $J_{1}^{\prime}(\lambda)>0$ and $\lim _{\lambda \rightarrow+\infty} J_{1}(\lambda)=+\infty$, so we see that there exists a unique $\lambda_{1}>0$ such that $J_{1}\left(\lambda_{1}\right)=0$ and for any $\lambda<\lambda_{1}, J_{1}(\lambda)<0$. Similarly, there exists another $\lambda_{2}>0$ such that $J_{2}\left(\lambda_{2}\right)=0$ and $J_{2}(\lambda)<0$ holds for any $\lambda<\lambda_{2}$. While $-\frac{\ln \kappa}{\tau}>0$ holds obviously, it must be true that $\lambda_{0}>0$.

For any $0<\bar{\lambda}<\lambda_{0}$, we will have $J_{1}(\bar{\lambda})<0, J_{2}(\bar{\lambda})<0$ and $\bar{\lambda}<-\frac{\ln \kappa}{\tau}$. Applying Itô's formula to $\mathrm{e}^{\bar{\lambda} t} V\left(\tilde{x}_{t}, r(t)\right)$, where $V$ is the Lyapunov function defined in (16), we find that

$$
\mathrm{e}^{\bar{\lambda} t} V\left(\tilde{x}_{t}, r(t)\right)=V\left(\tilde{x}_{0}, i_{0}\right)+\int_{0}^{t} \mathrm{e}^{\bar{\lambda} s}\left(\bar{\lambda} V\left(\tilde{x}_{s}, r(s)\right)+L V(x(s), x(s-\tau), s, r(s))\right) d s+M_{2}(t),
$$

where $M_{2}(t)$ is a local continuous martingale with the initial value $M_{2}(0)=0$.

Applying the methods used to estimate (36), the inequalities (4), (16) and (43) and the fact that

$$
\int_{0}^{t} \mathrm{e}^{\bar{\lambda} s}|x(s-\tau)|^{p} d s=\int_{-\tau}^{t-\tau} \mathrm{e}^{\bar{\lambda}(s+\tau)}|x(s)|^{p} d s \leqslant \mathrm{e}^{\bar{\lambda} \tau} \int_{-\tau}^{0}|x(s)|^{p} d s+\mathrm{e}^{\bar{\lambda} \tau} \int_{0}^{t}|x(s)|^{p} d s,
$$

it is true that

$$
\begin{aligned}
& \mathrm{e}^{\bar{\lambda} t} V\left(\tilde{x}_{t}, r(t)\right) \\
\leq & V\left(\tilde{x}_{0}, i_{0}\right)+\left(\bar{\lambda} c_{2}(1+\kappa)^{p-1}-(1-\delta(p-2))(1-\kappa)^{p-1}\right) \int_{0}^{t} \mathrm{e}^{\bar{\lambda} s}|x(s)|^{p} d s \\
& +\left(\bar{\lambda} c_{2}(1+\kappa)^{p-1} \kappa+\left(2 \delta+(1-\delta(p-2))(1-\kappa)^{p-1} \kappa\right) \int_{0}^{t} \mathrm{e}^{\bar{\lambda} s}|x(s-\tau)|^{p} d s\right. \\
& +\left(\bar{\lambda} c_{2}(1+\kappa)^{q-1}-\left(\beta-\frac{q-2}{q} \hat{\beta}\right)(1-\kappa)^{q-1}\right) \int_{0}^{t} \mathrm{e}^{\bar{\lambda} s}|x(s)|^{q} d s \\
& +\left(\bar{\lambda} c_{2}(1+\kappa)^{q-1} \kappa+\left(\frac{q-p+2}{q} \hat{\beta}+\left(\beta-\frac{q-2}{q} \hat{\beta}\right)(1-\kappa)^{p-1} \kappa\right)\right) \int_{0}^{t} \mathrm{e}^{\bar{\lambda} s}|x(s-\tau)|^{q} d s+M_{2}(t) \\
\leq & \zeta+J_{1}(\bar{\lambda}) \int_{0}^{t}|x(s)|^{p} d s+J_{2}(\bar{\lambda}) \int_{0}^{t}|x(s)|^{q} d s+M_{2}(t),
\end{aligned}
$$

where we define

$$
\begin{aligned}
\zeta= & c_{2}\left(2^{p-1}(1+\kappa)^{p}\|\xi\|^{p}+2^{q-1}(1+\kappa)^{q}\|\xi\|^{q}\right. \\
& +\mathrm{e}^{\bar{\lambda} \tau}\left(\bar{\lambda} c_{2}(1+\kappa)^{p-1} \kappa+(1-\delta(p-2))(1-\kappa)^{p-1} \kappa+2 \delta\right) \int_{-\tau}^{0}|x(s)|^{p} d s \\
& +\mathrm{e}^{\bar{\lambda} \tau}\left(\bar{\lambda} c_{2}(1+\kappa)^{q-1} \kappa+\frac{q-p+2}{q} \hat{\beta}+\left(\beta-\frac{q-2}{q} \hat{\beta}\right) \kappa(1-\kappa)^{q-1}\right) \int_{-\tau}^{0}|x(s)|^{q} d s .
\end{aligned}
$$

Taking the expectation of the localized form of $\mathrm{e}^{\bar{\lambda} t} V\left(\tilde{x}_{t}, r(t)\right)$ with the same stopping times $\tau_{\gamma}$ defined in (26), we see that

$$
\begin{aligned}
& E\left(e^{\bar{\lambda}\left(t \wedge \tau_{\gamma}\right)} V\left(\tilde{x}_{t \wedge \tau_{\gamma}}, r\left(t \wedge \tau_{\gamma}\right)\right)\right) \\
\leqslant & E\left(V\left(\tilde{x}_{0}, i_{0}\right)\right)+E \int_{0}^{t \wedge \tau_{\gamma}} \mathrm{e}^{\bar{\lambda} s}\left(\bar{\lambda} V\left(\tilde{x}_{s}, r(s)\right)+L V(x(s), x(s-\tau), s, r(s))\right) d s \\
\leqslant & K_{5}+J_{1}(\bar{\lambda}) E \int_{0}^{t \wedge \tau_{\gamma}} \mathrm{e}^{\bar{\lambda} s}|x(s)|^{p} d s+J_{2}(\bar{\lambda}) E \int_{0}^{t \wedge \tau_{\gamma}} \mathrm{e}^{\bar{\lambda} s}|x(s)|^{q} d s,
\end{aligned}
$$


where $K_{5}:=E \zeta$ is a constant.

Now from the facts that, $J_{1}(\bar{\lambda})<0, J_{2}(\bar{\lambda})<0$, and using (17), we can derive that

$$
c_{1} E\left(\mathrm{e}^{\bar{\lambda}\left(t \wedge \tau_{\gamma}\right)}\left|x\left(t \wedge \tau_{\gamma}\right)-G\left(x\left(t \wedge \tau_{\gamma}-\tau\right)\right)\right|^{p}\right) \leqslant K_{5} .
$$

Letting $\gamma \rightarrow+\infty$, we have

$$
\mathrm{e}^{\bar{\lambda} t} E|x(t)-G(x(t-\tau))|^{p} \leqslant \frac{K_{5}}{c_{1}} .
$$

In order to get the exponential stability of $E|x(t)|^{p}$, by setting $\theta=\frac{\kappa}{1-\kappa}$ in (3) and using the inequality $\bar{\lambda}<-\frac{\ln \kappa}{\tau}$, we can derive that

$$
\begin{aligned}
\mathrm{e}^{\bar{\lambda} t} E|x(t)|^{p} & \leqslant(1-\kappa)^{1-p} \mathrm{e}^{\bar{\lambda} t} E\left|\tilde{x}_{t}\right|^{p}+\kappa^{1-p} \mathrm{e}^{\bar{\lambda} t} E|G(x(t-\tau))|^{p} \\
& \leqslant \frac{K_{5}}{c_{1}}(1-\kappa)^{1-p}+\kappa \mathrm{e}^{\bar{\lambda} \tau}\left(\mathrm{e}^{\bar{\lambda}(t-\tau)} E|x(t-\tau)|^{p}\right)
\end{aligned}
$$

and then

$$
\sup _{0 \leqslant s \leqslant t} \mathrm{e}^{\bar{\lambda} s} E|x(s)|^{p} \leqslant \frac{K_{5}}{c_{1}}(1-\kappa)^{1-p}+\kappa \mathrm{e}^{\bar{\lambda} \tau} E\|\xi\|^{p}+\kappa \mathrm{e}^{\bar{\lambda} \tau} \sup _{0 \leqslant s \leqslant t} \mathrm{e}^{\bar{\lambda} s} E|x(s)|^{p} .
$$

Applying the fact that $\kappa \mathrm{e}^{\bar{\lambda} \tau}<1$, it is easy to get

$$
\sup _{0 \leqslant s \leqslant t} \mathrm{e}^{\bar{\lambda} s} E|x(s)|^{p} \leqslant \frac{1}{1-\kappa \mathrm{e}^{\bar{\lambda} \tau}}\left(\frac{K_{5}}{c_{1}}(1-\kappa)^{1-p}+\kappa \mathrm{e}^{\bar{\lambda} \tau} E\|\xi\|^{p}\right),
$$

which implies that

$$
\sup _{0 \leqslant t<+\infty} \mathrm{e}^{\bar{\lambda} t} E|x(t)|^{p} \leqslant \frac{1}{1-\kappa \mathrm{e}^{\bar{\lambda} \tau}}\left(\frac{K_{5}}{c_{1}}(1-\kappa)^{1-p}+\kappa \mathrm{e}^{\bar{\lambda} \tau} E\|\xi\|^{p}\right),
$$

and

$$
\limsup _{t \rightarrow \infty} \frac{1}{t} \log \left(E|x(t)|^{p}\right) \leqslant-\bar{\lambda} .
$$

Again applying $J_{1}(\bar{\lambda})<0$ and $J_{2}(\bar{\lambda})<0$ to $(51)$, we can obtain $\mathrm{e}^{\bar{\lambda} t}|x(t)-G(x(t-\tau))|^{p} \leqslant$ $\zeta+M_{2}(t)$. The non-negative semi-martingale convergence theorem yields

$$
\limsup _{t \rightarrow \infty}\left(\mathrm{e}^{\bar{\lambda} t}|x(t)-G(x(t-\tau))|^{p}\right)<\infty, \text { a.s. }
$$

Then there exists a finite positive random variable $\bar{\zeta}$ such that

$$
\sup _{0 \leqslant t<\infty}\left(\mathrm{e}^{\bar{\lambda} t}|x(t)-G(x(t-\tau))|\right) \leqslant \bar{\zeta}, \text { a.s. }
$$

Now by the same way as in (56), it holds that for any $t \geqslant 0$,

$$
\sup _{0 \leqslant s \leqslant t}\left(\mathrm{e}^{\bar{\lambda} s}|x(s)|^{p}\right) \leqslant \frac{1}{1-\kappa e^{\tau}}\left[(1-\kappa)^{1-p} \sup _{0 \leqslant s \leqslant t}\left(\mathrm{e}^{\bar{\lambda} s}|x(s)-G(x(s-\tau))|^{p}\right)+\kappa \mathrm{e}^{\tau}\|\xi\|\right] .
$$

Letting $t \rightarrow+\infty$ gives

$$
\sup _{0 \leqslant t \leqslant \infty}\left(\mathrm{e}^{\bar{\lambda} t}|x(t)|^{p}\right) \leqslant \frac{1}{1-\kappa e^{\tau}}\left((1-\kappa)^{1-p} \bar{\zeta}+\kappa \mathrm{e}^{\tau}\|\xi\|\right),
$$


and consequently

$$
\limsup _{t \rightarrow \infty} \frac{1}{t} \log \left(|x(t)| \leqslant-\frac{\bar{\lambda}}{p} .\right.
$$

Now from the arbitrary choice of $\bar{\lambda}$, we get the exponential stability of $E|x(t)|^{p}$ and $|x(t)|$ as required.

\section{Examples}

Example 5.1. Consider following one-dimensional hybrid NSDDE on $t \geqslant 0$

$$
d(x(t)-G(x(t-\tau)))=f(x(t), x(t-\tau), t, r(t)) d t+g(x(t-\tau), t, r(t)) d B(t),
$$

where $B(t)$ is a scalar Brownian motion and $r(t)$ is a Markov chain with state space $S=\{1,2,3,4\}$ with the generator

$$
\Gamma=\left[\begin{array}{cccc}
-4 & 1 & 2 & 1 \\
1 & -4 & 2 & 1 \\
2 & 1.8 & -4.8 & 1 \\
1 & 2 & 2 & -5
\end{array}\right] .
$$

Let $\tau=0.01, G(y)=0.1 y$ and the coefficients are defined by

$$
\begin{aligned}
& f(x, y, 1)=1-2 x+0.5 y, \quad g(y, 1)=0.2 y ; \quad f(x, y, 2)=1-3 x+0.7 y, \quad g(y, 2)=0.2 y \text {; } \\
& f(x, y, 3)=1-2 x^{3}, \quad g(y, 3)=0.4 y^{2} ; \quad f(x, y, 4)=1-3 x^{3}, \quad g(y, 4)=0.4 y^{2} .
\end{aligned}
$$

In this example, the system will satisfy the linear growth condition in mode 1 and mode 2 . But in mode 3 and mode 4 , the system has nonlinearities. The state space $S$ will be divided into $S_{1}=\{1,2\}$ and $S_{2}=\{3,4\}$. Also we have $\kappa=0.1$. Take $p=2, q=4$. Based on the inequalities $|a b| \leqslant \frac{1}{2}\left(a^{2}+b^{2}\right)$ and the Young's inequality, it is straightforward to show that condition (5) and (6) are satisfied with

$$
\begin{gathered}
(x-G(y)) f(x, y, 1)+\frac{3}{2}|g(y, 1)|^{2} \leqslant 0.5-(x-0.1 y)^{2}+0.105 y^{2} ; \\
(x-G(y)) f(x, y, 2)+\frac{3}{2}|g(y, 2)|^{2} \leqslant 0.5-2.48(x-0.1 y)^{2}+0.08 y^{2} ; \\
\quad(x-G(y)) f(x, y, 3)+\frac{1}{2}|g(y, 3)|^{2} \\
=\quad(x-0.1 y)-2(x-0.1 y)^{4}-0.6 y(x-0.1 y)^{3}-0.06 y^{2}(x-0.1 y)^{2}-0.002 y^{3}(x-0.1 y) \\
\leqslant 0.5+0.5(x-0.1 y)^{2}-1.565(x-0.1 y)^{4}+0.14 y^{4} ;
\end{gathered}
$$

and

$$
(x-G(y)) f(x, y, 4)+\frac{1}{2}|g(y, 4)|^{2} \leqslant 0.5+0.5(x-0.1 y)^{2}-2.27(x-0.1 y)^{4}+0.23 y^{4} .
$$


Meanwhile, using the same notations, as in Theorem 3.1, we can identify the following parameters:

$$
\begin{gathered}
\alpha_{11}=0.5, \alpha_{12}=-1, \alpha_{13}=0.105 \\
\alpha_{21}=0.5, \alpha_{22}=-2.48, \alpha_{23}=0.08 \\
\alpha_{31}=0.5, \alpha_{32}=0.5, \alpha_{33}=0, \alpha_{34}=1.565, \alpha_{35}=0.14 \\
\alpha_{41}=0.5, \alpha_{42}=0.5, \alpha_{43}=0, \alpha_{44}=2.27, \alpha_{35}=0.23
\end{gathered}
$$

and then

$$
\mathcal{A}=\left(\begin{array}{cccc}
6 & -1 & -2 & -1 \\
-1 & 8.48 & -2 & -1 \\
-2 & -1.8 & 3.8 & -1 \\
-1 & -2 & -2 & 4
\end{array}\right) \text { and } \mathcal{D}=\left(\begin{array}{cc}
8 & -1 \\
-1 & 13.92
\end{array}\right)
$$

Obviously, from

$$
\mathcal{A}^{-1}=\left(\begin{array}{cccc}
0.3985 & 0.2071 & 0.4588 & 0.2661 \\
0.1887 & 0.2584 & 0.3388 & 0.1965 \\
0.4032 & 0.3213 & 0.8519 & 0.3941 \\
0.3956 & 0.3417 & 0.7101 & 0.6118
\end{array}\right) \text { and } \mathcal{D}^{-1}=\left(\begin{array}{ll}
0.1261 & 0.0091 \\
0.0091 & 0.0725
\end{array}\right),
$$

we can see that $\mathcal{A}$ and $\mathcal{D}$ are nonsingular $M$-matrices. Simple calculations give

$$
\theta_{1}=1.3305, \theta_{2}=0.9824, \theta_{3}=1.9707, \theta_{4}=2.0592 .
$$

Let $\beta=3$ to get $\eta_{1}=0.4056$ and $\eta_{2}=0.2447$. The parameters in Theorem 3.1 are $\widehat{\beta}=0.9472$ and $\delta=0.1406$.

We can now verify that all conditions in Theorem 3.1 are satisfied and following boundedness of the solution can be obtained:

$$
\limsup _{t \rightarrow \infty} \frac{1}{t} \int_{0}^{t} E|x(s)|^{4} d s \leqslant K_{1},
$$

and

$$
\limsup _{t \rightarrow \infty} E|x(t)|^{2} \leqslant K_{2}
$$

where $K_{1}$ and $K_{2}$ are positive constants as depicted in (31) and (41), respectively.

Example 5.2. Consider another equation with the same form as equation (58) with different coefficients, where $G(y)=0.1 \sin y$,

$$
\Gamma=\left[\begin{array}{cccc}
-2.9 & 2.6 & 0.2 & 0.1 \\
2.2 & -2.4 & 0.1 & 0.1 \\
0.2 & 2.6 & -4 & 1.2 \\
3.1 & 1.8 & 1.9 & -6.8
\end{array}\right]
$$


and the coefficients are defined by

$$
\begin{array}{cccc}
f(x, y, 1)=-2 x+0.1 y, & g(y, 1)=0.1 x+0.2 y ; & f(x, y, 2)=-3 x+y, & g(y, 2)=0.5 y ; \\
f(x, y, 3)=0.5 x-2 x^{3}-0.1 y, & g(y, 3)=0.1 x+0.4 y^{2} ; & f(x, y, 4)=0.1 x-3 x^{3}-0.2 y, & g(y, 4)=0.4 y^{2} .
\end{array}
$$

As in the first example, we see different structures in $\{1,2\}$ and $\{3,4\}$. Again let $S_{1}=\{1,2\}$, $S_{2}=\{3,4\}$ and $p=2, q=4$. Applying the fact that $(a y+b \sin y)^{2} \leq(|a|+|b|)^{2} y^{2}$ and Young's inequality, conditions (5) and (6) are satisfied with

$$
\begin{gathered}
(x-G(y)) f(x, y, 1)+\frac{3}{2}|g(y, 1)|^{2} \leqslant-1.47(x-0.1 \sin y)^{2}+0.1773 y^{2} \\
(x-G(y)) f(x, y, 2)+\frac{3}{2}|g(y, 2)|^{2} \leqslant-2.5(x-0.1 \sin y)^{2}+0.97 y^{2} \\
(x-G(y)) f(x, y, 3)+\frac{1}{2}|g(y, 3)|^{2} \\
=0.005\left((x-0.1 \sin y)+\left(0.1 \sin y+4 y^{2}\right)\right)^{2}+0.5(x-0.1 \sin y)^{2}+(x-0.1 \sin y)(0.05 \sin y-0.1 y) \\
\leqslant-2(x-0.1 \sin y)((x-0.1 \sin y)+0.1 \sin y)^{3} \\
\leqslant \quad 0.51(x-0.1 \sin y)^{2}+0.01\left(0.1 \sin y+4 y^{2}\right)^{2}+(x-0.1 \sin y)(0.05 \sin y-0.1 y) \\
\leqslant-2(x-0.1 \sin y)^{4}-0.6(x-0.1 \sin y)^{3} \sin y-0.002(x-0.1 \sin y)(\sin y)^{3} \\
\leqslant \quad 0.56(x-0.1 \sin y)^{2}+0.1267 y^{2}-1.545(x-0.1 \sin y)^{4}+0.4715 y^{4}
\end{gathered}
$$

and

$$
(x-G(y)) f(x, y, 4)+\frac{1}{2}|g(y, 4)|^{2} \leqslant 0.15(x-0.1 \sin y)^{2}+0.225 y^{2}-2.32(x-0.1 \sin y)^{4}+0.36 y^{4} .
$$

For any $i \in S$, we have $\alpha_{i 1}=0$. The other parameters are

$$
\begin{gathered}
\alpha_{12}=-1.47, \alpha_{13}=0.1773 ; \alpha_{22}=-2.5, \alpha_{23}=0.97 ; \\
\alpha_{32}=0.56, \alpha_{33}=0.1267, \alpha_{34}=1.545, \alpha_{35}=0.4715 ; \\
\alpha_{42}=0.15, \alpha_{43}=0.225, \alpha_{44}=2.365, \alpha_{45}=0.315 ;
\end{gathered}
$$

and then

$$
\mathcal{A}=\left(\begin{array}{cccc}
5.84 & -2.6 & -0.2 & -0.1 \\
-2.2 & 7.4 & -0.1 & -0.1 \\
-0.2 & -2.6 & 2.82 & -1.2 \\
-3.1 & -1.8 & -1.9 & 6.5
\end{array}\right) \text { and } \mathcal{D}=\left(\begin{array}{cc}
8.82 & -2.6 \\
-2.2 & 12.4
\end{array}\right)
$$

From

$$
\mathcal{A}^{-1}=\left(\begin{array}{cccc}
0.2083 & 0.0837 & 0.0237 & 0.0089 \\
0.0660 & 0.1650 & 0.0148 & 0.0063 \\
0.1435 & 0.2222 & 0.4299 & 0.0850 \\
0.1596 & 0.1505 & 0.1411 & 0.1847
\end{array}\right) \text { and } \mathcal{D}^{-1}=\left(\begin{array}{cc}
0.1196 & 0.0251 \\
0.0212 & 0.0851
\end{array}\right),
$$


we can see that $\mathcal{A}$ and $\mathcal{D}$ are nonsingular $M$-matrices, and subsequently

$$
\theta_{1}=0.3245, \theta_{2}=0.2521, \theta_{3}=0.8806, \theta_{4}=0.6358
$$

Set $\beta=2$ to get $\eta_{1}=0.2894$ and $\eta_{2}=0.2126$. The parameters in Theorem 3.1 are $c_{2}=0.8806$, $\kappa=0.1, \widehat{\beta}=0.8304$ and $\delta=0.2445$. It is easily to check that all conditions in Theorem 3.1 and Theorem 4.3 are satisfied.

Solve equations in (48) to get $\lambda_{1}=0.2995, \lambda_{2}=0.1612$ and then $\lambda_{0}=0.1612$. Therefore, the equation is exponentially stable with

$$
\limsup _{t \rightarrow \infty} \frac{1}{t} \log \left(E|x(t)|^{2}\right) \leqslant-0.1612
$$

and

$$
\limsup _{t \rightarrow \infty} \frac{1}{t} \log (|x(t)|) \leqslant-0.0806 .
$$

The simulations of the sample paths of the Markov chain and the solution of this example are presented in Figure 1.

Example 5.3. As an application of Theorem 4.3, in this example, we will discuss the problem of robust exponential stability of a stable HNSDDE.

Use the same notation as in Assumption 3.1. For any $(x, t, i) \in \mathbb{R}^{n} \times \mathbb{R}_{+} \times S$, the function $F(x, t, i)$ satisfies

$$
x^{T} F(x, t, i) \leqslant\left\{\begin{array}{lll}
a_{i 1}|x|^{2}, & \text { if } & i \in S_{1} \\
a_{i 1}|x|^{2}-a_{i 2}|x|^{q-p+2}, & \text { if } & i \in S_{2}
\end{array}\right.
$$

where $a_{i 1}<0$ for $i \in S_{1}$ and $a_{i 2}>0$ for any $i \in S_{2}$. Also assume

$$
\mathcal{A}:=-\operatorname{diag}\left(p a_{11}, \cdots, p a_{N 1}\right)-\Gamma
$$

is a nonsingular $M$-matrix. It can be shown directly that following NSDDE

$$
d(x(t)-G(x(t-\tau)))=F(x(t)-G(x(t-\tau)), t, r(t)) d t
$$

is exponentially stable in the $p$-th moment for any initial data.

Suppose that the original stable equation (59) is subject to perturbations and the perturbed equation is

$$
d(x(t)-G(x(t-\tau)))=F(x(t)-G(x(t-\tau)), t, r(t)) d t+H(x(t), x(t-\tau), t, r(t)) d B_{t}
$$

with $H$ controlled by

$$
|H(x, y, t, i)|^{2} \leqslant \begin{cases}\bar{a}_{i}|y|^{2}, & \text { if } i \in S_{1} \\ \bar{a}_{i}\left(|x|^{q-p+2}+|y|^{q-p+2}\right), & \text { if } \quad i \in S_{2}\end{cases}
$$



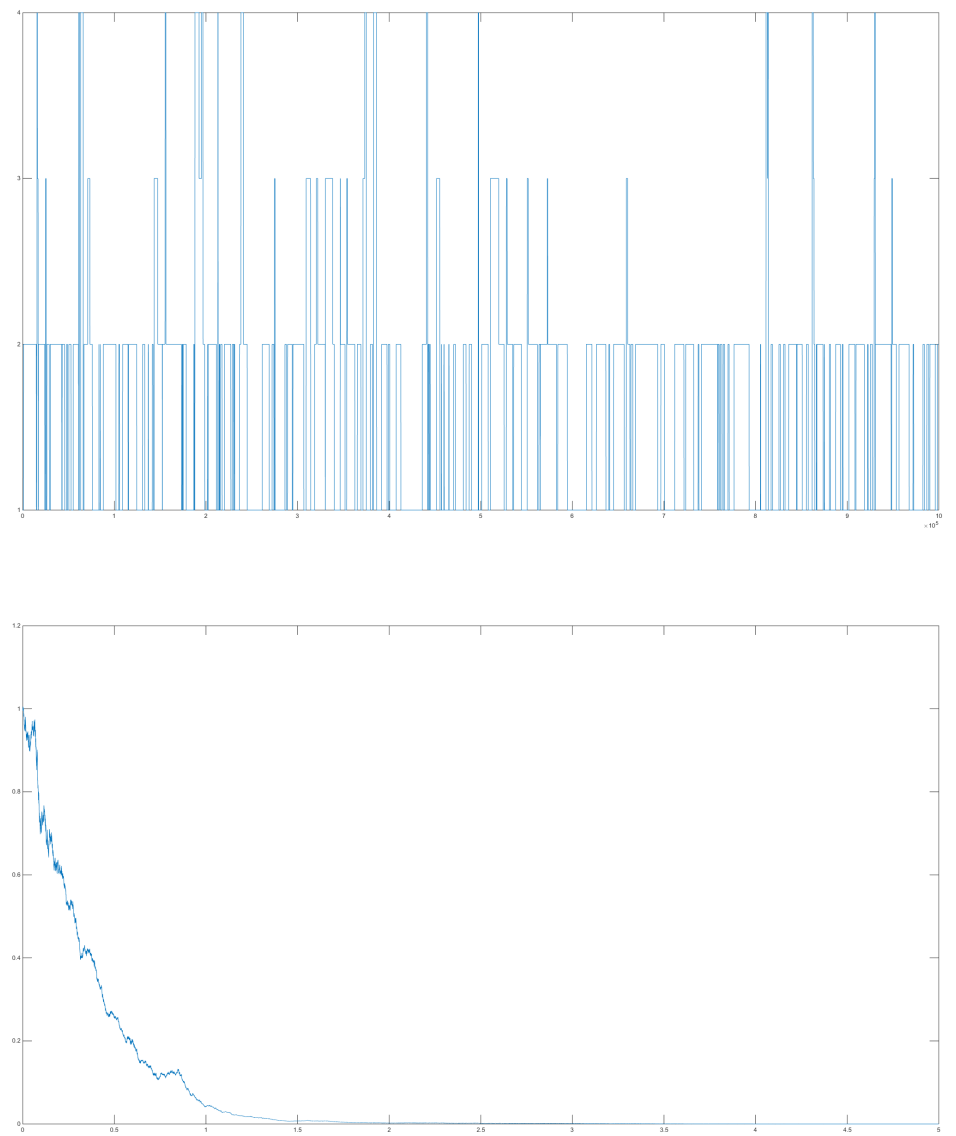

Figure 1: The sample path of the Markov chain and the solution with the initial data $\xi=\{\cos t: t \in$ $[-\tau, 0]\}$.

We will discuss the bound on $\bar{a}_{i}$ such that equation (60) remains exponentially stable.

Following the discussion in Theorem 3.1 and 4.3, we only need to verify conditions in (11), (12) and (13). If $i \in S_{1}$,

$$
(x-G(y))^{T} F(x-G(y))+\frac{q-1}{2}|H(x, y, t, i)|^{2} \leqslant a_{i 1}|x-G(y)|^{2}+\bar{a}_{i}|y|^{2} ;
$$

while if $i \in S_{2}$,

$$
\begin{aligned}
& (x-G(y))^{T} F(x-G(y))+\frac{p-1}{2}|H(x, y, t, i)|^{2} \\
\leqslant & a_{i 1}|x-G(y)|^{2}-\left(a_{i 2}-\frac{p-1}{2} \bar{a}_{i}(1+\kappa)^{q-p+1}\right)|x-G(y)|^{q-p+2}+\bar{a}_{i}\left(\frac{p-1}{2}(1+\kappa)^{q-p+1}+1\right)|y|^{q-p+2} ;
\end{aligned}
$$

Obviously,

$$
\mathcal{D}:=-\operatorname{diag}\left(q a_{11}, \cdots, q a_{N_{1}, 1}\right)-\left(\gamma_{i j}\right)_{i, j \in S_{1}}
$$

is a nonsingular $M$-matrix because all $a_{i 1}, i \in S_{1}$ are positive as noted in [22]. 
Applying the result in Remark 4.4 of [22], denote $\hat{a}$ be the maximum of the row sums of $D^{-1}$ and $\hat{b}=\max _{i \in S_{2}}\left(\sum_{j \in S_{1}} \gamma_{i j}\right)$. Fix $\beta$ a positive number less than $\frac{1}{1+\hat{a} \hat{b}} \min _{i \in S_{2}}\left\{p \theta_{i} a_{i 2}\right\}$, with $\theta$ defined by (9), and calculate $\eta$ as in (10). It can be easily shown that for any $i \in S_{2}$,

$$
p a_{i 2} \theta_{i}-\sum_{j \in S_{1}} \gamma_{i j} \eta_{j}>\beta .
$$

Now in order that the perturbed equation (60) remains exponentially stable, following conditions should be satisfied:

$$
\begin{aligned}
& \text { for any } i \in S_{1}, \bar{a}_{i} \theta_{i}<\frac{(1-\kappa)^{p}}{(p-2)(1-\kappa)^{p}+2} ; \\
& \text { for any } i \in S_{1}, q \bar{a}_{i} \eta_{i}<\frac{q(1-\kappa)^{q}}{(q-p+2)+(q-2)(1-\kappa)^{q}} \beta ; \\
& \text { for any } i \in S_{2}, p \bar{a}_{i}\left(\frac{p-1}{2}(1+\kappa)^{q-p+1}\right) \theta_{i}<\frac{q(1-\kappa)^{q}}{(q-p+2)+(q-2)(1-\kappa)^{q}} \beta \\
& \text { for any } i \in S_{2}, \frac{p-1}{2} \bar{a}_{i}(1+\kappa)^{q-p+1}<p a_{i 2} \theta_{i}-\sum_{j \in S_{1}} \gamma_{i j} \eta_{j}-\beta .
\end{aligned}
$$

Consequently, we have the bounds for $\bar{a}_{i}, i \in S$ such that the perturbed equation remains exponentially stable

$$
\bar{a}_{i}< \begin{cases}\min \left(\frac{(1-\kappa)^{p}}{\left((p-2)(1-\kappa)^{p}+2\right) \theta_{i}}, \frac{q(1-\kappa)^{q}}{\left((q-p+2)+(q-2)(1-\kappa)^{q}\right) q \eta_{i}} \beta\right), & \text { if } i \in S_{1} \\ \min \left(\frac{\beta}{p\left(\frac{p-1}{2}(1+\kappa)^{q-p+1}\right)} \frac{q(1-\kappa)^{q}}{(q-p+2)+(q-2)(1-\kappa)^{q}}, \frac{2\left(p a_{i 2} \theta_{i}-\sum_{j \in S_{1}} \gamma_{i j} \eta_{j}-\beta\right)}{(p-1)(1+\kappa)^{q-p+1}}\right), & \text { if } i \in S_{2} .\end{cases}
$$

\section{Conclusion}

In this article, we study existence and properties of the solution for a class of highly nonlinear hybrid neutral stochastic differential delay equations, whose coefficients have different nonlinear structures in different switching modes. Applying $M$-matrix techniques, we have proposed suitable conditions on coefficients to get the existence and uniqueness of the solution. We have also proved some typical properties of the solution, including its boundedness and asymptotic stability. Under these conditions, the solution will be exponentially stable in $p$-th moment and almost surely. Also there are some pending problems to be solved in future discussion, for example, controller design for stabilization of an unstable neutral system with highly nonlinear coefficients, delay-dependent criteria for exponential stability, etc.

\section{Acknowledgements}

The authors would like to thank the financial supports from the Natural Science Foundation of Shanghai(17ZR1401300). The research of X. Mao was supported by the Leverhulme Trust (RF2015-385), the Royal Society (WM160014, Royal Society Wolfson Research Merit Award). The authors wish to thanks two anonymous reviewers for their careful reading and valuable comments. The comments have helped to improve the quality of this article. 


\section{References}

[1] Y. Kuang, Delay Differential Equations with Applications in Population Dynamics, Academic Press, San Diego, 1993.

[2] V.B. Kolmanovskii, A.D. Myshkis, Applied Theory of Functional Differential Equations, Kluwer Academic, Dordrecht, 1992.

[3] R.K. Brayton, Bifurcation of periodic solutions in a nonlinear difference-differential equation of neutral type, Quart. Appl. Math. 24 (1966) 215-224.

[4] X. Mao, Stochastic Differential Equations and Applications, Horwood, Chichester, UK, 2007.

[5] X. Mao, Y. Shen, C. Yuan, Almost surely asymptotic stability of neutral stochastic differential delay equations with Markovian switching, Stoch. Proc. Appl. 118 (2008) 1385-1406

[6] Q. Luo, X. Mao, Y. Shen, New criteria on exponential stability of neutral stochastic differential delay equations, Syst. Contr. Lett. 55(10)(2006) 826-834.

[7] J. Luo, Fixed points and stability of neutral stochastic delay differential equations, J. Math. Anal. Appl. 334(2007) 431-440.

[8] J. Bao, Z. Hou. C. Yuan, Stability in distribution of neutral stochastic differential delay equations with Markovian switching, Stat. Prob. Letter. 79(2009) 1663-1673.

[9] X. Li, X. Mao, A note on almost sure asymptotic stability of neutral stochastic delay differential equations with Markovian switching, Automat. 48(9)(2012) 2329-2334.

[10] B. Song, J. Park, Z. Wu, Y. Zhang, New results on delay-dependent stability analysis for neutral stochastic delay systems, J. Franklin Inst., 50(4)(2013) 840-852.

[11] Y. Xu, Z. He, Exponential stability of neutral stochastic delay differential equations with Markovian switching, Appl. Math. Letter. 52(2016) 64-73.

[12] X. Zong, F. Wu, Exponential stability of the exact and numerical solutions for neutral stochastic delay differential equations, Appl. Math. Model. 1(2016) 19-30.

[13] L. Hu, X. Mao, L. Zhang, Robust stability and boundedness of nonlinear hybrid stochastic differential delay equations, IEEE Trans. Automa. Control. 58(2013) 2319-2332.

[14] M. Milošević, Highly nonlinear neutral stochastic differential equations with time-dependent delay and the Euler-Maruyama method, Math. Comput. Modell. 54(9-10)(2011), 2235-2251. 
[15] M. Milošević, Almost sure exponential stability of solutions to highly nonlinear neutral stochastic differential equations with time-dependent delay and the Euler-Maruyama approximation, Math. Comput. Modell. 57(3-4)(2013), 887-899.

[16] M. Milošević, Convergence and almost sure exponential stability of implicit numerical methods for a class of highly nonlinear neutral stochastic differential equations with constant delay, J. Comput. Appl. Math. 280(2015) 248-264.

[17] Y. Xie, C. Zhang, Asymptotical boundedness and moment exponential stability for stochastic neutral differential equations with time-variable delay and markovian switching, Appl. Math. Lett. 70(2017) 46-51.

[18] M. Li, F. Deng, Almost sure stability with general decay rate of neutral stochastic delayed hybrid systems with Lèvy noise, Nonlin. Anal. Hybr. Syst. 24(2017) 171-185.

[19] W. Fei, L. Hu, X. Mao, M. Shen, Delay Dependent Stability of Highly Nonlinear Hybrid Stochastic Systems, Automat. 82(2017) 165-170.

[20] M. Shen, W. Fei, X. Mao, Y. Liang, Stability of highly nonlinear neutral stochastic differential delay equations, Syst. Contr. Lett. 115(2018) 1-8.

[21] X. Zong, F. Wu, C. Huang, The Boundedness and Exponential Stability Criterions for Nonlinear Hybrid Neutral Stochastic Functional Differential Equations, Abstr. Appl. Anal. 2013, Article ID 138031, 12 pages.

[22] W. Fei, L. Hu, X. Mao, M. Shen, Structured Robust Stability and Boundedness of Nonlinear Hybrid Delay Systems, SIAM J. Contr. Optim. 56(4)(2018) 2662-2689.

[23] X. Mao, C. Yuan, Stochastic Differential Equations with Markovian Switching, Imperial College Press, 2006. 